\title{
ESCUELAS DE CAMPO DE AGRICULTORES DE Theobroma cacao L. EN EL BAJO CAGUAN (Experiencia, Resultados y Lecciones Aprendidas)
}

\author{
Verenice Sánchez Castillo ${ }^{1}$ \\ Jean Alexander Gamboa Tabares ${ }^{2}$
}

Recibido el 30 de abril de 2013 y aprobado el 5 de julio de 2013

\section{RESUMEN}

Localización. La Región del Bajo Caguán se encuentra localizada en las riberas del río Cagúan, jurisdicción del municipio de Cartagena del Chaira, suroriente del Departamento del Caquetá en la región Amazónica Colombiana. Objetivo. Compartir los resultados metodológicos y de formación logrados mediante las Escuelas de Campo de Agricultores de Theobroma cacao L. desarrollados por la Universidad de la Amazonia en el marco del proyecto: "Desempeño agronómico, evaluación fitosanitaria y caracterización molecular de clones promisorios de Theobroma cacao $L$. provenientes de materiales vegetales élite presentes en sistemas productivos del bajo Caguán, municipio de Cartagena del Chairá" (ECAs-CAGUAN) que contó con el apoyo de Colciencias, el Comité de Cacaoteros del Bajo Caguán y Suncillas "Chocaguán" y la Parroquia San Isidro Labrador. Metodología. El trabajo se realizó a partir del aprendizaje por descubrimiento, partiendo de los conocimientos y la experiencia previa de los campesinos, la reflexión, la nueva información y la aplicación de la misma. Esta situación implicó la formación a profesionales como facilitadores de ECAs, la definición participativa del currículo, la construcción de protocolos y la aplicación flexible de estos en campo. Resultados. La ECAs - CAGUAN, en su primer ciclo de formación lograron formar a 43 cultivadores y productores de Theobroma cacao $\mathrm{L}$, es decir que el $78 \%$ de los que iniciaron el proceso y se logró un Indice de Incremento del Conocimiento (IIC) del 34\%. Una de las lecciones aprendidas y quizás la más importante es la necesidad que las ECAs incorporen otros elementos de trabajo que van más allá de lo técnico y está relacionado con estrategias de trabajo social, afectivo y de sensibilidad, esto contribuirá a mejorar los procesos de adopción tecnológica en campo. Conclusiones. El proceso de las ECAs- Caguan, permitió a los agricultores entre otras cosas entender que el hallazgo de los élites y/o el mejoramiento genético es solo un factor que ayudará a que su producto mejore, pero la mayor responsabilidad está precisamente en ellos mismos, en la realización de las prácticas culturales y de manejo de sus cultivos.

Palabras clave: ECAs, facilitador, agricultor 


\title{
Theobroma cacao L. FARMER FIELD SCHOOLS IN LOWER CAGUAN (Experience, Results and Lessons Learned)
}

\begin{abstract}
Location. The Bajo Caguan region is located on the banks of the Cagúan River, municipality of Cartagena del Chaira, southeast of the Department of Caquetá in the Colombian Amazon region. Objective.To share methodological and training results achieved through Theobroma cacao L. Farmer Field Schools developed by the University of the Amazon under the project: " Agronomic performance, phytosanitary evaluation and molecular characterization of promising Theobroma cacao L. clones from elite plant material present in productive systems in the Bajo Caguan municipality of Cartagena del Chairá" (RCT-Cagúan) that was supported by Colciencias, the Cocoa Committee of Bajo Caguan and Suncillas, "Chocaguán",and the San Isidro Labrador Parish. Methodology. The work was conducted from discovery learning, based on the farmers' previous knowledge and experience, reflection, new information and its application. This involved training professionals as FFS facilitators, the participatory definition of curriculum, building of protocols and flexible application of these in the field. Results. The FFS - Cagúan, in its first training cycle managed to train 43 farmers and producers of Theobroma cacao L, which means that $78 \%$ of those who started the process achieved an increase of Knowledge Index (IIC) of $34 \%$. One of the lessons learned and perhaps the most important one, is the need to incorporate to the FFS other work items that go beyond the technical and that are related to social work, affective and sensitivity strategies, which will help improve technological adoption processes in the field. Conclusions. The FFS-Caguan process, allowed farmers, among other things, to understand that finding the elites and/or genetic improvement is only one factor that will help improve their product, but the main responsibility lies precisely in themselves, in conducting cultural practices and crop management.
\end{abstract}

\section{Keywords:}

FFSs, facilitator, farmer

\section{INTRODUCCIÓN}

El Theobroma cacao L., llegó al Caguán como cultivo de importancia económica, a finales de los 80's, esta zona por tradición extractivista y luego cocalera, había sido militarizada, lo que impedía el libre comercio de la pasta de coca, situación que fue aprovechada por el sacerdote de Remolinos del Caguán, Padre Jacinto Franzoi, de la Parroquia San Isidro Labrador para impulsar una campaña en pro de los cultivos lícitos, esta se llamó: "no a la 
coca, si al caucho y al cacao; con esta iniciativa organizaciones italianas y la parroquia, buscaban que las familias sembraran Theobroma cacao L. y dejaran los cultivos de uso ilícito. Algunos campesinos creyeron en la alternativa e iniciaron aunque con poco conocimiento, las siembras de cacao. Posteriormente conformaron el Comité de Cacaoteros de Remolinos del Caguán y Suncillas (CHOCAGUÁN) y con el apoyo de Naciones Unidas construyeron la planta de chocolate "CHOCAGUAN" dando un mayor valor agregado a su producto, por esta lucha, en el año 2004 se hicieron acreedores al Premio Nacional de Paz.

Desde entonces y aunque se rescatan logros significativos de los cultivadores de cacao, también es necesario precisar que las actividades económico - productivas de este colectivo, se ha debatido entre vaivenes y altibajos, pues si bien los campesinos hicieron un tránsito de la coca al cacao, en especial por los problemas de inseguridad social de lo ilícito, que hicieron que éste fuera cada vez menos atractivo y que buscaran nuevas alternativas productivas, algunos reincidieron en los cultivos de uso ilícito y abandonaron el cacao, esto colocó y aún coloca en riesgo el proceso social del cacao alternativa socioeconómica.

Alguna de las razones y quizás la más importante por la cual las familias reinciden en lo ilícito, están relacionadas con su baja competitividad ante el mercado, por sus pocos niveles de producción, sus plantaciones son en un 90\% híbridas, nunca se les hizo mejoramiento genético, por tanto tienen una alta susceptibilidad a plagas y enfermedades, esto aunado a la falta de conocimiento acerca del manejo cultural, ha hecho que los problemas fitosanitarios invadan los cultivos y sean cada vez menos productivos. Otro tema que afecta los niveles de producción es precisamente la avanzada edad de los sembrados, pues algunos superan los 20 años de edad, durante los cuales no se realizaron acciones para su sostenimiento y hoy la mejor opción es la renovación y/o el establecimiento de nuevas plantaciones

No obstante, ciertas familias han iniciado ya con el establecimiento de nuevas plantaciones, pasando de las hibridas a plantaciones clonales, sin embargo, este proceso se ha adelantado con materiales introducidos, cuyo comportamiento no ha sido evaluado en la zona, por tanto se requiere generar conocimiento alrededor de posibles materiales élite que permita mejorar las condiciones de los sembrados y por ende la producción.

El proyecto "Desempeño agronómico, evaluación fitosanitaria y caracterización molecular de clones promisorios de Theobroma cacao $L$. provenientes de materiales vegetales élite presentes en sistemas productivos del bajo Caguán, municipio de Cartagena del Chairá" nace de esta necesidad, fue formulado por la Universidad de la Amazonia en alianza con CHOCAGUÁN, con el ánimo de encontrar materiales locales resistentes a plagas y enfermedades, superiores en productividad a los materiales híbridos cultivados tradicionalmente, a partir de la determinación de bases científicas sólidas que permitan a la organización comunitaria, impulsar la renovación de las plantaciones viejas y el fomento de plantaciones 
nuevas de cacao, en aras de una economía lícita para los habitantes de la zona.

La estrategia para la generación de este nuevo conocimiento o eje transversal del proyecto consiste precisamente en un proceso de formación local dirigido a la gestión de la investigación, alrededor del establecimiento de Escuelas de Campo de Agricultores ECAs (ECA-CAGUÁN), como ejercicio piloto dentro de la Cadena Productiva de Cacao del Departamento del Caquetá. Las ECAs, constituyen un enfoque metodológico que busca fortalecer el conocimiento técnico de un cultivo en particular, partiendo del diálogo entre campesinos y técnicos; en ellas, los temas curriculares no son prediseñados, sino que se definen con la comunidad, no es condicionante para ingresar saber leer y escribir pues la metodología es lúdica y con demostración de método, tienen un proceso y un fin dado por el ciclo del cultivo que se trabaje y las reglas para su funcionamiento se constituyen en colectivo. En las sesiones de trabajo en cada uno de los temas, que además son seleccionados por los campesinos, de acuerdo a sus necesidades, se parte de los conocimientos previos de los sujetos y se incita al autodescubrimiento, a través de: 1) la experiencia, 2) reflexión, 3) nueva información, y 4) aplicación. Al finalizar un ciclo de ECAs, Ios asistentes son evaluados y certificados de acuerdo a los resultados de conocimiento adquiridos (Ardon, 2003).

En este sentido, más que a sesiones de capacitación, con las ECAs se le apunta entonces a un proceso educativo, partiendo de que la educación de acuerdo con Ludojoski (1972), es algo intencional del ser humano en desarrollo, que en teoría contribuye al perfeccionamiento de su personalidad teniendo como base el relacionamiento y dialogo con: 1) la historia, en el sentido de las características propias de cada época y lo que ellas representan; 2) la naturaleza, entendida como factor hereditario y el medio ambiente físico; y 3) la cultura y desde ella los valores y creencias que las personas construyen, utilizan y apropian durante siglos.

Ahora, si bien era un proceso educativo, recordar que tenía una particularidad y es que era un proceso educativo con adultos en Escuelas de Campo de Agricultores, por ello el trabajo se apoyó en la teoría de la andragogía, que desde el enfoque de la educación permanente, considera que el logro del proceso con adultos solo es posible si se crea una metodología especial, adecuada a la situación propia particular del adulto y el educando como colectivo, esto amerita evaluar el grado de madurez de su personalidad, la capacidad intelectual, pero sobre todo sus conocimientos teóricos y prácticos (Ludojoski, 1972).

A partir de lo anterior, se plantea otro reto y es el tema "didáctico"; pues es el responsable de conducir el proceso de aprendizaje al ritmo particular de los adultos así como sus intereses y motivaciones. De igual forma el esquema grupos que contribuyan a una formación integradora de los valores individuales y comunitarios del grupo. 


\section{MATERIALES Y MÉTODOS}

\section{Localización geográfica y temporal:}

El departamento del Caquetá está ubicado al sur de Colombia y se sitúa entre los $2^{\circ} 58$ minutos de latitud Norte y $0^{\circ} 40$ minutos de latitud Sur, entre los $71^{\circ} 30$ minutos y $76^{\circ} 15$ minutos de longitud al oeste del Greenwich (IGAC, 1993).

El Municipio de Cartagena del Chairá está localizado a la margen derecha del Río Caguán, aguas abajo del Sur del Departamento, sobre las Coordenadas $01^{\circ} 21^{\prime} 00^{\prime \prime}$ de Latitud Norte y $74^{\circ} 50^{\prime} 24^{\prime \prime}$ de Longitud Oeste del Meridiano de Greenwich. Se encuentra a una distancia de 120,5 Km de la capital del departamento, Florencia. El municipio presenta una precipitación promedio anual entre 2500 y $3500 \mathrm{~mm}$, distribuidos en 10 meses, con una época relativamente seca entre diciembre y marzo; humedad relativa cercana al punto de saturación, con un promedio del $90 \%$; temperatura media de $26^{\circ} \mathrm{C}$; evaporación de 970,5 mm/año; brillo solar de 1508 horas luz/año; velocidad del viento promedio de 1,3 m/seg. y altura promedio de 234 m.s.n.m.

El establecimiento de las ECAs, se hizo en el área rural del municipio de Cartagena del Chairá, en veredas localizadas sobre el margen del río Caguán, que corresponde a la zona de acción del proyecto de la Universidad-Chocaguan-Colciencias. Si bien el proyecto contemplaba el establecimiento de una ECA, debido al alto número de interesados, amplio nivel de dispersión y teniendo en cuenta los altos costos de transporte fluvial en que tendrían que incurrir las familias interesadas, se decidió establecer tres ECAs en tres puntos de encuentro que coincidieron con los lugares donde se establecerían las parcelas de investigación del proyecto: La vereda Cuba, La Vereda Palmichales y el caserío Cristales.

La metodología de trabajo fue la siguiente:

\section{Establecimiento de las ECAs}

Para el establecimiento de las ECAs, se siguieron las pautas metodológicas propuestas por Gallager (1999) y adaptadas por Pumisacho et al (2005). De igual forma se revisaron las experiencias en este campo en países como Nicaragua a través del Programa Estatal para la Seguridad Alimentaria (FAO, 2005); la experiencia en cacao desarrollada por el IICA en cacao en Perú (2007); por el Centro Internacional de la Papa (CIP), en papa en la ciudad de Andahuylas-Perú (CIP, 2008); el trabajo de USAID a través del programa MIDAS en Colombia en cultivos como Banano, Cacao y Hortalizas (USAID, 2009) y las experiencias en café y cacao desarrolladas por el IICA en la Selva Central (IICA, 2010) entre otras. 
- Definición del grupo: a través de las directivas de CHOCAGUÁN, se hizo una convocatoria abierta para las personas interesadas en participar en la Escuela de Campo de agricultores de cacao en la zona de estudio. En dicho encuentro se socializó el esquema metodológico de las ECAs, los compromisos y requisitos que se adquirían al ingresar a ellas.

- Caracterización del perfil del grupo Con los interesados, se hizo la caracterización de los intereses del grupo y se definieron las reglas de juego para el funcionamiento. Para ello, se elaboró un cuadro de doble entrada, donde en la primera columna se registró el nombre de los participantes, en frente de cada nombre se colocó el número de árboles de cacao, la edad y la producción. Así mismo, se consignaron las expectativas en relación al proceso de capacitación-formación.

- Definición del contenido temático: con cada uno de los grupos establecidos, se desarrolló un taller mediante la técnica de lluvia de ideas, en la que los cacaocultores listaron las necesidades de capacitación que a su juicio requerían. Posteriormente, se hicieron pruebas de caja para identificar el nivel de conocimiento, las claridades y confusiones que se podrían tener alrededor de las temáticas técnicas generales alrededor del cultivo. Finalmente y teniendo en cuenta los resultados de lo que consideraban que requerían aprender y los resultados de la prueba de caja, se obtuvo un listado amplio de los temas y con los integrantes de la ECA en pleno, se hizo respectiva selección en orden de prioridad empleando una matriz de priorización.

\section{Construcción de las Guías de trabajo protocolos}

Con anterioridad a esta parte se hizo el entrenamiento del equipo de investigación en metodología ECA. Para ello se realizó una gira de aprendizaje al departamento de Santander con los facilitadores de FEDECACAO, donde se pudo apreciar en campo la ejecución de una ECA. El objetivo fue que el equipo no fuera un transferente de tecnología sino que se convirtiera en un facilitador del proceso.

Aunque la palabra "protocolo", lingüísticamente hace alusión a modelos y pasos a seguir de manera estricta, lo que aquí se elaboró fue un modelo de lo que se pudiera trabajar en cada uno de los temas, sin ser una camisa de fuerza. Para ello, el equipo técnico y metodológico del proyecto se reunió y 
para cada tema identificó unas preguntas introductorias clave, las dinámicas, las prácticas de campo a realizar, las evaluaciones del aprendizaje y también los requerimientos logísticos y de materiales necesarios para cada sección.

\section{Desarrollo de actividades de aprendizaje}

Teniendo como guía los protocolos diseñados en el apartado 2, se desarrollaron por espacio de un año de manera práctica los contenidos temáticos para la ECA seleccionados por los productores. Para ello se hicieron encuentros mensuales con duración de ocho horas, entre conversatorio y práctica con tareas particulares asignadas para el periodo entre una y otra sesión.

\section{Evaluación}

Se hicieron dos tipos de evaluación: evaluación de aprendizaje y las lecciones aprendidas de las ECAS. La primera evaluación siguiendo las recomendaciones propuestas por USAID (2009), se hicieron al finalizar cada uno de los encuentros y consistió en una prueba escrita diseñada con preguntas, dibujos y ejercicios de campo los cuales se aplicaban en la ECA siguiente al tema evaluado. Al tener todos los resultados, estos fueron ponderados y expresados en porcentajes y de manera comparativa con los resultados de la prueba de caja inicial se halló el Índice de Conocimiento (IC). Las lecciones aprendidas se obtuvieron a partir de diálogos reflexivos con los campesinos acerca del proceso, en talleres de campo, la información fue sistematizada en una plantilla de doble entrada con el ánimo de poder identificar consensos y casos particulares.

\section{RESULTADOS Y DISCUSIÓN}

\section{Establecimiento de las ECA}

\section{Definición del grupo}

Se instalaron las tres Escuelas de Campo en los puntos definidos en la metodología: ECA Caguán - Cuba: 15 inscritos; ECA CaguánPalmichales: 10 inscritos y ECA Caguán - Cristales: 30 inscritos. Aunque las ECAs se establecieron en lugares y con grupos diferentes, los acuerdos internos y los compromisos a los que se llegaron para el funcionamiento fueron similares: 1) se recibirían inscripciones tanto de gente que tenía cacao, como que quisiera sembrar. 2) el cacaotero que faltara a más de dos encuentros ya no podría nivelarse y perdería el ciclo. 3) cada integrante llevaría algo 
para preparar el almuerzo comunitario, con excepción de Cristales, pues allá el centro educativo a través del aporte del Centro y de los padres ofertarían el almuerzo para los estudiantes de la ECA. 4) la periodicidad de los encuentros sería mensual con fechas definidas en el encuentro anterior y solo por motivos de fuerza mayor se podría modificar y 5) las prácticas de campo se harían tanto en las parcelas de investigación como en las de los productores.

\section{Caracterización del perfil de los grupos}

Se encontraron tres tipos de asistentes a las ECAs. El primer grupo denominado Cacao en producción, que representó el $49 \%$ de los asistentes a las ECAs, presentándose su mayor presencia en la ECACAGUAN- Cuba, con 12 productores, seguido de Cristales y finalmente Palmichales (Figura 1). Se caracterizaban por tener plantaciones que en promedio, no llegaban a los 400 árboles en producción. Las edades de estas plantaciones superaban los 10 años de edad y su manejo se limitaba a recolectar los frutos, el rendimiento promedio se halló en 0,3 Kg.ha.año. Este grupo aunque correspondía a los productores expresaron su interés de establecer cacao nuevo.

El segundo tipo de asistentes fueron los interesados en cultivar, que presentó el $40 \%$, implicaban familias que habían tenido cultivo y lo habían dejado perder, o agricultores que querían dejar la coca e iniciar con el cultivo de cacao, la mayor presencia de asistentes con estas características se tuvo en la ECACAGUAN-Cristales. Finalmente y un grupo más pequeño, con el 11\%, lo conformaban agricultores con cacao en crecimiento, esta personas con esfuerzos y el apoyo de instituciones como la Cruz Roja Internacional, habían iniciado el establecimiento de nuevas plantaciones pero se habían quedado sin asistencia técnica (Gráfica 1):

\section{Gráfica 1.Tipología de asistente a las ECAs}

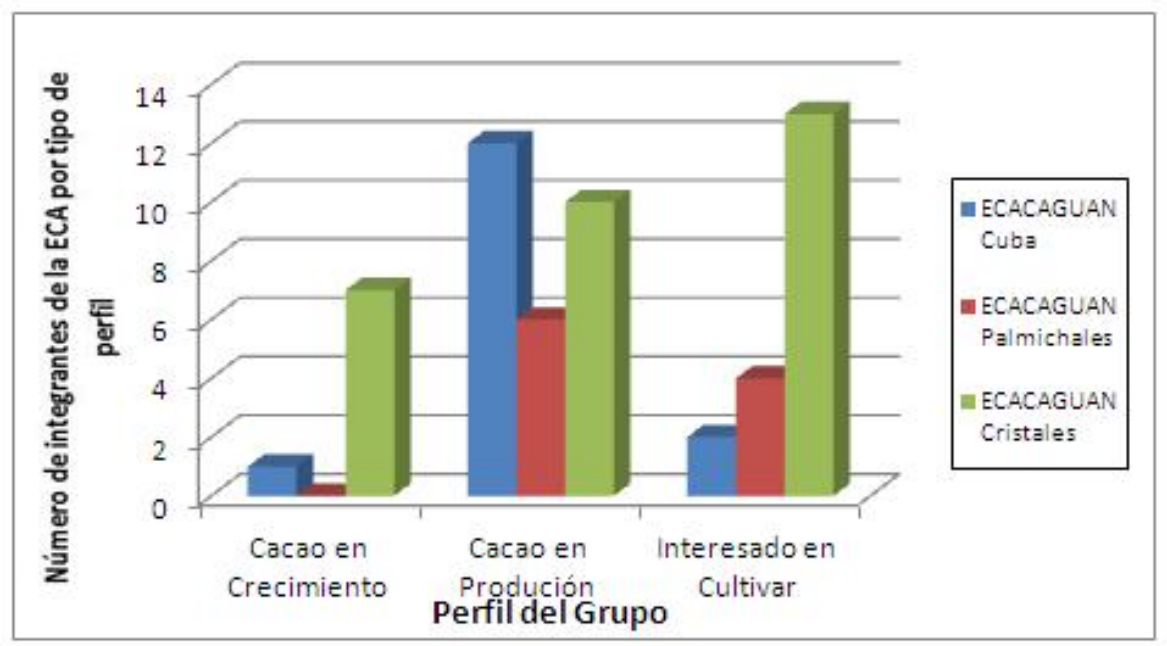




\section{Definición del contenido temático}

Identificación de intereses de capacitación: la lluvia de ideas permitió identificar un interés marcado hacia la parte de injertación y mejoramiento genético del con el $94 \%$, siendo este el tema que aparecía con mayor frecuencia en los tres puntos de encuentro; seguido de intereses como fertilización y manejo del suelo: $89 \%$, siembra: $70 \%$, y en menor frecuencia manejo de plagas: $40 \%$ enfermedades: $38 \%$ y prácticas de cosecha y poscosecha del grano: $18 \%$ (cuadro 1):

Cuadro 1. Frecuencia de necesidades de capacitación ECAs - Caguán

\begin{tabular}{|l|c|c|c|c|}
\hline $\begin{array}{l}\text { Necesidades } \\
\text { identificadas por } \\
\text { los asistentes a } \\
\text { las Ecas }\end{array}$ & $\begin{array}{l}\text { ECACAGUAN } \\
\text { Cuba }\end{array}$ & $\begin{array}{l}\text { ECACAGUAN } \\
\text { Palmichales }\end{array}$ & $\begin{array}{l}\text { ECACAGUAN } \\
\text { Cristales }\end{array}$ & FRECUENCIA \\
\hline Injertación & 25 & 10 & 17 & 52 \\
\hline $\begin{array}{l}\text { Fertilización/manejo } \\
\text { del suelo }\end{array}$ & 28 & 7 & 14 & 49 \\
\hline Siembra & 20 & 9 & 10 & 39 \\
\hline Manejo de plagas & 7 & 9 & 6 & 22 \\
\hline Enfermedades & 8 & 6 & 7 & 21 \\
\hline $\begin{array}{l}\text { Cosecha y } \\
\text { poscosecha }\end{array}$ & 3 & 4 & 3 & 10 \\
\hline
\end{tabular}

Pruebas de caja: los conocimientos técnicos que de manera general tenían los campesinos alrededor del cultivo del cacao - en todas la ECAs-Caguán- coincidían en claridades y confusiones (Cuadro 2): 
Cuadro 2. Resultados prueba de caja en las ECAs-CAGUAN

\begin{tabular}{|c|c|c|c|c|c|c|c|c|}
\hline \multirow[t]{2}{*}{ PREGUNTA PRUEBA DE CAJA } & \multicolumn{2}{|c|}{ CUBA } & \multicolumn{2}{|c|}{$\begin{array}{c}\text { PAL } \\
\text { MIC } \\
\text { HAL } \\
\text { ES }\end{array}$} & \multicolumn{2}{|c|}{$\begin{array}{l}\text { CRIS } \\
\text { TAL }\end{array}$} & \multicolumn{2}{|c|}{$\begin{array}{c}\text { TOTA } \\
\text { LES } \\
\text { ITEM } \\
\text { A } \\
\end{array}$} \\
\hline & $\mathrm{C}$ & I & C & I & $\mathrm{C}$ & I & $\mathrm{C}$ & I \\
\hline $\begin{array}{l}\text { Qué ataque de plagas identifica en estos } \\
\text { frutos de cacao }\end{array}$ & 1 & $\begin{array}{l}1 \\
4\end{array}$ & 3 & 7 & 5 & 25 & 9 & 46 \\
\hline ¿Cuál es la importancia de la injertación? & 1 & $\begin{array}{l}1 \\
4 \\
\end{array}$ & 4 & 6 & 10 & 20 & 15 & 40 \\
\hline $\begin{array}{l}\text { Qué enfermedades identifica en estos } \\
\text { frutos }\end{array}$ & 2 & $\begin{array}{l}1 \\
3\end{array}$ & 5 & 5 & 8 & 22 & 15 & 40 \\
\hline $\begin{array}{l}\text { Qué enfermedades identifica en estos } \\
\text { frutos }\end{array}$ & 2 & $\begin{array}{l}1 \\
3\end{array}$ & 4 & 6 & 10 & 20 & 16 & 39 \\
\hline $\begin{array}{l}\text { Cuál es la densidad de siembra ideal para } \\
\text { el cultivo del cacao }\end{array}$ & 8 & 7 & 3 & 7 & 6 & 24 & 17 & 38 \\
\hline Tipo de suelo requerido para el cacao & 5 & $\begin{array}{l}1 \\
0\end{array}$ & 5 & 5 & 7 & 23 & 17 & 38 \\
\hline ¿Cuál es la importancia de un vivero? & 5 & $\begin{array}{l}1 \\
0\end{array}$ & 3 & 7 & 11 & 19 & 19 & 36 \\
\hline $\begin{array}{l}\text { Cuál es la profunidad efectiva requerida } \\
\text { para el cultivo del cacao }\end{array}$ & 4 & $\begin{array}{l}1 \\
1\end{array}$ & 1 & 9 & 14 & 16 & 19 & 36 \\
\hline Cuándo se realizan las podas & 5 & $\begin{array}{l}1 \\
0\end{array}$ & 2 & 8 & 12 & 18 & 19 & 36 \\
\hline $\begin{array}{l}\text { ¿Qué paisajes es mejor para el cultivo del } \\
\text { cacao? }\end{array}$ & 8 & 7 & 5 & 5 & 7 & 23 & 20 & 35 \\
\hline $\begin{array}{l}\text { Qué plagas del cacao identifica en este } \\
\text { insectario }\end{array}$ & 5 & $\begin{array}{l}1 \\
0\end{array}$ & 3 & 7 & 12 & 18 & 20 & 35 \\
\hline ¿Qué es injertacion? & 5 & $\begin{array}{l}1 \\
0\end{array}$ & 5 & 5 & 12 & 18 & 22 & 33 \\
\hline $\begin{array}{l}\text { ¿Cuáles son las prácticas culturales para el } \\
\text { mantenimiento del cultico de cacao? }\end{array}$ & 7 & 8 & 5 & 5 & 11 & 19 & 23 & 32 \\
\hline Tipos de podas que se realizan al cacao & 4 & $\begin{array}{l}1 \\
1\end{array}$ & 4 & 6 & 16 & 14 & 24 & 31 \\
\hline TOTAL & 62 & $\begin{array}{c}14 \\
8\end{array}$ & $\begin{array}{l}5 \\
2\end{array}$ & $\begin{array}{l}8 \\
8\end{array}$ & $\begin{array}{c}14 \\
1\end{array}$ & $\begin{array}{c}27 \\
9\end{array}$ & $\begin{array}{c}25 \\
5\end{array}$ & $\begin{array}{c}51 \\
5\end{array}$ \\
\hline
\end{tabular}

- De las 770 respuestas efectuadas por los participantes, el 67\% fueron incorrectas y el 33\% correctas. De las 515 respuestas incorrectas, se detectó que la mayor confusión estuvo en el tema de plagas enfermedades con el $16 \%$, dicha confusión en relación al diagnóstico de plantaciones, puso en evidencia por tanto también errores en su forma de manejo.

En segundo lugar de preguntas con respuesta incorrecta, se hallaron las relacionadas con el tema de suelos, injertación y siembras-viveros, con el 14\% cada una. En una menor proporción de respuestas incorrectas el tema de podas con el 13\%; selección de terrenos con el $7 \%$ y prácticas culturales con el $6 \%$. Cabe advertir sin embargo que estos dos últimos temas solo tuvieron una pregunta referida a ellos y esto pudo afectar su porcentaje de participación en lo incorrecto.

Esta prueba de caja, también permitió identificar el desconocimiento y por ende la no aplicación de las prácticas culturales preventivas y de manejo cultural en los cultivos (6\%), tema que extrañamente nunca apareció como un interés de capacitación, lo que dejaba en claro la poca importancia en él, que sin embargo era el causante de 
los mayores problemas fitosanitarios y reproductivos en el cultivo. De igual forma se apreció una gran diferencia entre las formas de pensar entre productores con relación a las distancias y densidades de siembra y tipos de paisaje requeridos para el cultivo, pues mientras que para algunos los terrenos más óptimos eran las vegas, otros consideraban que en las vegas el cacao se inundaba y se perdía, por haber más humedad (Gráfica 2):

Gráfica 2. Porcentaje de respuestas incorrectas por temas abordados en la prueba de caja en las ECAs - Caguan

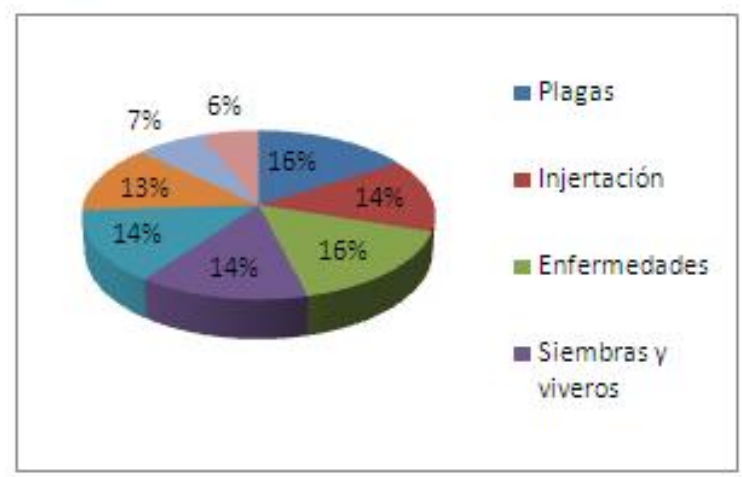

Matriz de priorización: en las ECAs-Caguán de manera general se definieron los siguientes temas en el orden de prioridad como se puede seguir:

Encuentro 1: Manejo semilla asexual de Theobroma cacao L. Encuentro 2. Establecimiento de viveros. Encuentro 3. Identificación y manejo de plagas y enfermedades en vivero.

Encuentro 4. Selección de terrenos para Theobroma cacao L. y análisis de suelos. Encuentro 5. Trazado, ahoyado y siembra de Theobroma cacao L. en agroforestería. Encuentro 6. Manejo del cultivo del plátano como componente del SAF con Theobroma cacao L. Encuentro 7. Injertación en Theobroma cacao L. Encuentro 8. Podas de formación y manejo en Theobroma cacao L.

\section{Construcción de las guías de trabajo: protocolos}

Aunque inicialmente se había dicho que se diseñarían los nueve protocolos para cada encuentro, finalmente se diseñaron 20, teniendo en cuenta otros posibles temas que en la realización de las ECAs, los productores fueron identificando como importantes para un segundo nivel de formación. Por el volumen de los protocolos aquí solo se presentará qué lleva cada uno de ellos, para mayor información de estos se invita a consultar el libro "Establecimiento y sostenimiento de plantaciones de Theobroma cacao L. en sistemas agroforestales: un currículo de acción participativa para el autoaprendizaje en la ECA-CAGUAN". Las guías de trabajo o protocolos contienen una serie de recomendaciones para facilitar una ECA en cada uno de los temas, estas servirán de apoyo para cualquier otra ECA de Theobroma cacao L. que se quiera 
desarrollar en otro proyecto o espacio, no pretende ser una camisa de fuerza. Cada una de las 20 guías o protocolos contemplan dos apartados generales: A y $\mathrm{B}$ :

A. Preparación del encuentro: 1) reflexiones del equipo facilitador antes del encuentro, 2) se dan las indicaciones para seleccionar los escenarios de capacitación, las recomendaciones para preparar los lugares con los materiales y las herramientas necesarias para las actividades, y 3) se indica qué tipo de material pedagógico se requiere para el trabajo, así como la forma de diseñar las ayudas pictóricas y las preguntas.

B. Desarrollo del encuentro: cada protocolo incluye 1) una dinámica introductoria sugerida alusiva al Theobroma cacao L. y a la zona de estudio, 2) dinámica de formación de grupos, construida a partir del contexto Caguán, 3) las preguntas introductorias sugeridas para el tema de cada encuentro, 4) dinámica de reanimación luego del almuerzo, 5) indicaciones para la práctica de campo, 6) esquema metodológico para hacer la plenaria de los resultados, y 7) sugerencias para hacer la evaluación de la sección y el cierre.

\section{Desarrollo de actividades de aprendizaje}

En cada uno de los encuentros realizados, se pudo encontrar una amplia gama de conocimientos previos, algunos no tan confiables "científicamente hablando", pero que fueron igualmente discutidos y evaluados en las actividades de aprendizaje con el ánimo de llegar a un conocimiento validado y construido dentro de la ECA.

Durante el proceso en las ECAs - Caguán, se realizaron 20 encuentros. En cuanto a la temática abordada, se tiene que en las ECA Caguán Cristales y Palmichales se lograron desarrollar todos los temas del primer nivel, es decir fase de establecimiento y manejo, encuentros del número 1 al 7; en la ECA Caguán-Cuba por motivos de orden público se hicieron desde los temas 1 al 4 . Se contó con la asistencia permanente de en promedio de 55 personas por ECA, con una interesante participación de los jóvenes y niños, en especial de la ECA Caguán- Cristales, pues de las 55 personas que asistieron regularmente a las ECAs Caguán, 28 se encontraron en rangos de edades entre los 10 y 25 años de edad.

En relación a los resultados encontrados en cada uno de los encuentros y por lo que implica en términos de espacio en el presente escrito, solo se resumirán algunos de los principales hallazgos en los respectivos temas (cuadro 3): 
Cuadro 3. Resultados ECAs

\begin{tabular}{|c|c|c|}
\hline TEMA & $\begin{array}{l}\text { ACTIVIDADES } \\
\text { REALIZADAS }\end{array}$ & $\begin{array}{l}\text { PRINCIPALES RESULTADOS } \\
\text { TECNICOS }\end{array}$ \\
\hline \multirow{4}{*}{$\begin{array}{l}\text { Manejo de } \\
\text { semilla } \\
\text { asexual }\end{array}$} & Selección de mazorcas & \multirow{4}{*}{$\begin{array}{l}\text { Se establecieron comparaciones } \\
\text { entre los granos de las mazorcas } \\
\text { hibridas y las del patrón universal } \\
\text { IMC } 67 \text {, identificando mayor número } \\
\text { de granos, mayor tamaño y mejor } \\
\text { desprendimiento del mucílago en las } \\
\text { de IMC } 67 \text {. }\end{array}$} \\
\hline & Selección de semilla & \\
\hline & $\begin{array}{|ll|}\begin{array}{l}\text { Proceso de limpieza y } \\
\text { desinfección }\end{array} & \\
\end{array}$ & \\
\hline & Pregerminación & \\
\hline \multirow{5}{*}{ Viveros } & $\begin{array}{l}\text { Diseño y medidas de un } \\
\text { vivero }\end{array}$ & \multirow{5}{*}{$\begin{array}{l}\text { Se construyeron tres viveros con } \\
\text { capacidad para } 5000 \text { plántulas en } \\
\text { cada uno de los puntos de } \\
\text { encuentro de las ECAs. La mayor } \\
\text { dificultad estuvo en la consecución } \\
\text { de la materia orgánica, suplir esta } \\
\text { dificultad se crearon estrategias para } \\
\text { que la gente fuera haciéndolo de } \\
\text { manera periódica con suficiente } \\
\text { anticipación al montaje de los } \\
\text { viveros. }\end{array}$} \\
\hline & $\begin{array}{l}\text { Construcción de la } \\
\text { estructura }\end{array}$ & \\
\hline & Preparaciór & \\
\hline & Embo & \\
\hline & Sier & \\
\hline \multirow{8}{*}{$\begin{array}{l}\text { Manejo de } \\
\text { Plagas y } \\
\text { enfermed } \\
\text { ades en } \\
\text { vivero }\end{array}$} & i de daños & \multirow{8}{*}{$\begin{array}{l}\text { Las plagas identificadas en los tres } \\
\text { viveros fueron mariquitas, grillos y } \\
\text { gusanos. El control se hizo de } \\
\text { manera orgánica con purin de ajo } \\
\text { con aji y extracto de fique con } \\
\text { aplicaciones día por medio. De igual } \\
\text { forma se identificaron enfermedades } \\
\text { como Phytoptora y Antracnosis, para } \\
\text { ello se hicieron aplicaciones de } \\
\text { caldo sulfocálcico, como el problema } \\
\text { persistía se hicieron aplicaciones de } \\
\text { un fungicida orgánico de una casa } \\
\text { comercial }\end{array}$} \\
\hline & $\begin{array}{l}\text { Identificación del agente } \\
\text { causal }\end{array}$ & \\
\hline & $\begin{array}{l}\text { aración de purines y } \\
\text { ctos para el control }\end{array}$ & \\
\hline & Aplicación, & \\
\hline & $\begin{array}{l}\text { Iden } \\
\text { de } \mathrm{e}\end{array}$ & \\
\hline & $\begin{array}{ll}\text { iento del } \\
\text { ausal }\end{array}$ & \\
\hline & $\begin{array}{l}\text { aración de fungicidas } \\
\text { nicos }\end{array}$ & \\
\hline & & \\
\hline \multirow{5}{*}{$\begin{array}{l}\text { Caracteriz } \\
\text { ación } \\
\text { biofísica y } \\
\text { química } \\
\text { del suelo }\end{array}$} & $\begin{array}{l}\text { Toma de muestras de } \\
\text { suelo }\end{array}$ & \multirow{5}{*}{$\begin{array}{l}\text { Suelos de vega: horizonte A: } 7 \mathrm{~cm} \text {; } \\
\text { profundidad efectiva: } 1.20 \quad \mathrm{~cm} \text {; } \\
\text { penetrabilidad: } 0,092 \mathrm{MGP} \text {; PH: } 4,5 \text {; } \\
\text { Organismos vivos identificados: } \\
\text { lombrices, cienpies, cucarrones, } \\
\text { grillos. Suelos de lomerio: Ha: } 1 \mathrm{~cm} \text {; } \\
\text { profundidad efectiva: } 1,30 \quad \mathrm{~m} \text {; } \\
\text { penetrabilidad: } 560 \quad \mathrm{~N} \quad \mathrm{PH} 2,8 \text {; } \\
\text { Organismos identificados: cienpies }\end{array}$} \\
\hline & $\begin{array}{l}\text { Criterios para seleccionar } \\
\text { un suelo de cacao }\end{array}$ & \\
\hline & $\begin{array}{l}\text { Identificación } \\
\text { organismos vivos } \\
\end{array}$ & \\
\hline & \begin{tabular}{|l}
$\begin{array}{l}\text { Análisis del perfil de } \\
\text { suelos }\end{array}$ \\
\end{tabular} & \\
\hline & & \\
\hline
\end{tabular}




\begin{tabular}{|c|c|c|}
\hline & \begin{tabular}{|l|} 
Prueba del penetrometro \\
Identificación r de \\
deficiencias nutricionales \\
en el cultivo
\end{tabular} & \\
\hline \multirow{5}{*}{$\begin{array}{l}\text { Trazado } \\
\text { de un } \\
\text { cultivo de } \\
\text { cacao en } \\
\text { SAF y } \\
\text { Agoforest } \\
\text { ería }\end{array}$} & Selección de terreno & \multirow{5}{*}{$\begin{array}{l}\text { Tres terrenos seleccionados, } \\
\text { adecuados y con trazado para } \\
\text { plantaciones de cacao en } \\
\text { monocultivo y Agroforestería en } \\
\text { diferentes distancias de siembra. } \\
\text { Para el ejercicio de campo de la } \\
\text { ECA se estableció una parcela con } \\
\text { distancias de } 3,5 \mathrm{~m} \times 3,5 \mathrm{~m} \text { de } \\
\text { Cacao en Agroforestería con } \\
\text { plátano. }\end{array}$} \\
\hline & Adecuación & \\
\hline & $\begin{array}{l}\text { Trazado cacao } \\
\text { monocultivo y saf }\end{array}$ & \\
\hline & Distancias de siembra & \\
\hline & Ahoyado & \\
\hline \multirow{6}{*}{$\begin{array}{l}\text { Trazado } \\
\text { ahoyado y } \\
\text { siembra } \\
\text { del } \\
\text { componen } \\
\text { te Plátano } \\
\text { en el SAF } \\
\text { con } \\
\text { Cacao }\end{array}$} & $\begin{array}{l}\text { Selección y desinfección } \\
\text { del colino }\end{array}$ & \multirow{6}{*}{$\begin{array}{l}100 \text { Colinos seleccionados, } \\
\text { desinfectados y establecidos por las } \\
\text { ECAs en los puntos de encuentro de } \\
\text { Palmichales y en Cristales, como } \\
\text { producto de la práctica de campo. } \\
\text { Se identificó la presencia de plagas } \\
\text { como el picudo y enfermedades } \\
\text { como la Sigatoka negra y el } \\
\text { Maduraviche, se aplicaron los } \\
\text { principales correctivos. }\end{array}$} \\
\hline & Ahoyado & \\
\hline & Encalado & \\
\hline & Siembra & \\
\hline & $\begin{array}{l}\text { Labores culturales } \\
\text { mantenimiento }\end{array}$ & \\
\hline & $\begin{array}{l}\text { Plagas y enfe } \\
\text { en plantaciones a }\end{array}$ & \\
\hline \multirow{6}{*}{ Injertacion } & $\begin{array}{l}\text { Identificación de patrones } \\
\text { y clones }\end{array}$ & \multirow{6}{*}{$\begin{array}{l}\text { Se injertaron plántulas tanto en } \\
\text { vivero como en sitio definitivo. } \\
\text { Plantaciones hibridas con } 15 \text { años } \\
\text { de producción, fueron injertadas por } \\
\text { sus propios dueños, asistentes a las } \\
\text { ECAs. El porcentaje de prendimiento } \\
\text { fue del } 10 \% \text { en razón a la falta de } \\
\text { práctica de los participantes. }\end{array}$} \\
\hline & Selección de baretas & \\
\hline & Selección de yema & \\
\hline & Tipos de injertación & \\
\hline & Amarre y & \\
\hline & $\begin{array}{l}\text { ado y v } \\
\text { ndimiento }\end{array}$ & \\
\hline \multirow{6}{*}{$\begin{array}{l}\text { Podas en } \\
\text { Cacao }\end{array}$} & Tipos de poda & \multirow{6}{*}{$\begin{array}{l}\text { Plantaciones de cacao con más de } \\
10 \text { años de edad, fueron podadas y } \\
\text { rehabilitadas por los estudiantes de } \\
\text { las ECAs. A algunas se les } \\
\text { manejaron los chupones para hacer } \\
\text { rehabilitación }\end{array}$} \\
\hline & $\begin{array}{l}\text { Tiempos y factores de la } \\
\text { poda }\end{array}$ & \\
\hline & Herramientas & \\
\hline & Realización de podas & \\
\hline & $\begin{array}{l}\text { Elaboración de pastas } \\
\text { cicatrizantes }\end{array}$ & \\
\hline & & \\
\hline
\end{tabular}

En el cuadro 3, se resumen de manera general los principales resultados de los encuentros en la ECAs Caguan, cabe mencionar que estas ECAs obedeciendo los requerimientos de los asistentes y el perfil del grupo, iniciaron el proceso de formación con el tema de selección de la semilla y el establecimiento de los viveros. Esta situación es bastante particular, pues de acuerdo a las experiencias por ejemplo en Papa en la ECAs realizadas por el Centro Internacional de la Papa-CIP (2009), en Café por el Internacional Coffee Partner -ICP (2006), en Cacao por el IICA en Perú (2007), se tiene que las ECAs generalmente inician su proceso de formación a partir del tema plagas y enfermedades; además algunas se dedican puntualmente a una plaga o enfermedad, generalmente no se aborda desde el proceso de siembra de la plantación. 
La anterior particularidad puede tener su explicación en dos líneas, teniendo en cuenta que ECA es un mundo diferente y no sería posible establecer generalidades: una es en la genealogía de la ECA como proceso surgido de una necesidad y para atender un problema, el cual es identificado sobre lo que ya está, en este caso la plantación y otra es el perfil del grupo que se trabaje. A diferencia de los ejemplos referidos y en la misma línea de la experiencia de Pumishaco et al (2005), en agroforestería, el enfoque de las ECAs Caguán considera necesario brindar herramienta para aquellos agricultores que ya sea por decisión propia o porque su plantación lo amerita establecerán nuevas plantaciones, no solo solución de problemas particulares sino ofrecer nuevas alternativas.

Otras experiencias por su parte inician desde el Diagnóstico Rural Participativo, vinculando herramientas como el diagrama de Ven o diagnóstico de necesidades de la comunidad (IICA, 2007), al respecto se tiene que este tipo de trabajos generan un mayor nivel de confianza entre el equipo facilitador del proceso, pero debe advertirse hasta donde se compromete la ECA, pues no se debe olvidar la razón de ser de esta.

De acuerdo a los estudios encontrados, un ciclo de ECAs tiene una duración entre 10 y 12 sesiones, sin embargo, se reconocen la necesidad de que el ciclo sea más amplio. IICA (2006), plantea que un ciclo de formación debe ser de aproximadamente 23 sesiones de trabajo, distribuido en 4 módulos generales: lo técnico en establecimiento de plantación, manejo del cultivo, agroindustria y evaluación y entrega de resultados, este planteamiento y forma de desarrollar las ECAs, coincide en cierta medida con lo realizado en las ECAs en el Caguán donde en siete encuentros se abordó el proceso de establecimiento y renovación teniendo en cuenta el perfil de los agricultores que asistían a las mismas; pero teniendo claro que es un primer nivel de aproximación, y que aún queda pendiente el manejo integral del cultivo y la agroindustria. Sin embargo a partir de la dinámica de los ejercicios se pudo identificar que para lograr mayor apropiación de los temas, aún solo para establecimiento y rehabilitación se requieren más de las ocho sesiones realizadas.

\section{Evaluación}

\section{Evaluación del aprendizaje}

El tema de mayor apropiación de conceptos y manejo en campo fue el de trazado, ahoyado y siembra del cultivo del cacao donde el $91 \%$ de los agricultores demostraron claridad y domino; seguidamente el tema de podas con el $81 \%$, plátano y suelos con el $60 \%$ e injertación y viveros con el $52 \%$ (Gráfica 3). El aspecto de plagas y enfermedades, continua siendo una dificultad para los agricultores pues aunque en el inicio en la prueba de caja el $72 \%$ de ellos tuvieron respuestas no acertadas en relación al tema, aún después del proceso de formación en las ECAs, el $50 \%$ de los asistentes continuaron con dificultades en el tema de identificación de los daños por plagas y síntomas de la enfermedad. Cabe mencionar que estos porcentajes son relativos a la población asistente a los encuentros que si bien en las pruebas de caja el total fue de 55 agricultores, este número varió en cada encuentro (Gráfica 3): 
Gráfica 3. Prueba de caja

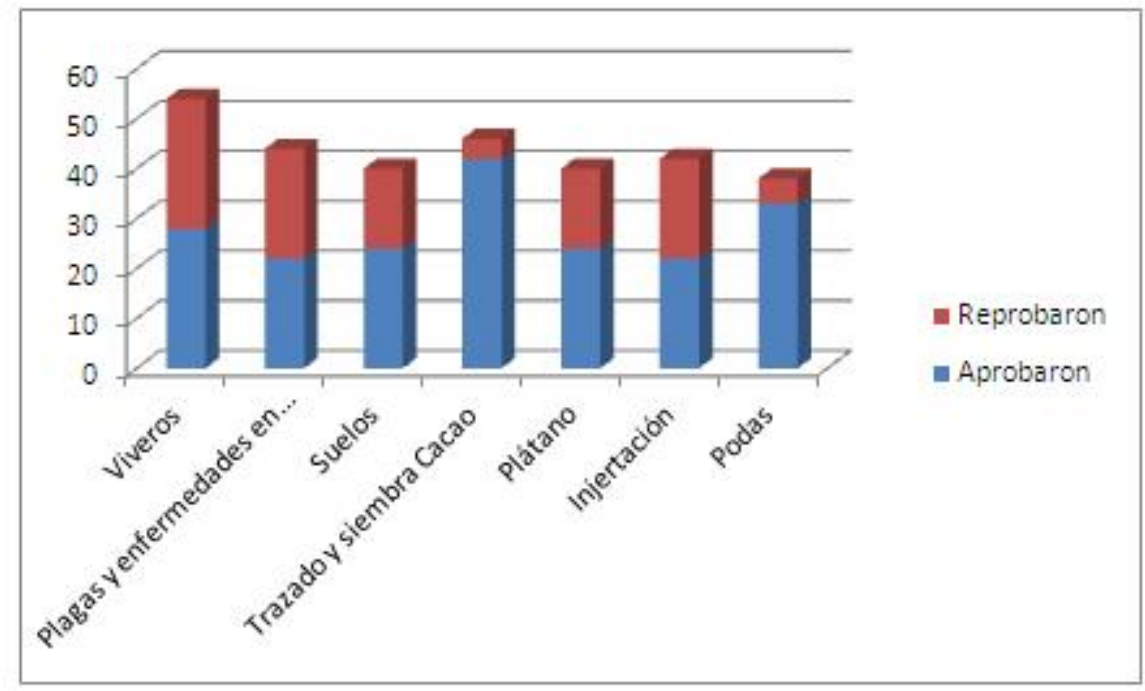

Se aplicaron siete pruebas de conocimiento a 43 agricultores en las ECAs de Cristales y Palmichales, cada prueba en promedio tuvo entre seis y siete preguntas, para un total de 1.698 preguntas realizadas, de las cuales 1140 fueron respondidas de manera acertada, es decir el $67 \%$ y las 558 restantes presentaron respuestas incorrectas (33\%). Lo anterior dejó ver un Índice de Incremento del Conocimiento (ICC) del 34\%, teniendo en cuenta el $33 \%$ del conocimiento inicial registrado por los participantes. Este resultado aunque positivo, pues se logró incrementar el conocimiento de los productores casi al doble, muestra que es necesario continuar con el trabajo pues aún se tiene un 33\% de conocimientos que no están claros y que ponen en riesgo el mantenimiento de las plantaciones.

En términos generales, se tiene que la existencia de adecuados conocimientos previos en algunos temas, aunada a la validación de recomendaciones técnicas mediante los ejercicios prácticos realizados en cada finca, facilitó la adopción de estos por parte de los agricultores.

De manera particular se tiene que el tema "podas de manejo" que aunque medianamente se conocía, no se realizaban en las plantaciones de los campesinos, se realizó en tres plantaciones antiguas (una por cada ECA), este ejercicio permitió visibilizar un impacto positivo en el cultivo en relación a la entrada de luz en el cultivo, coloración del follaje, desarrollo de la plantación, floración y fructificación. Cabe mencionar que en los inicios, los agricultores, se mostraron escépticos hacia la práctica, pues a su modo inicial de ver "cortarle las ramas al árbol de cacao era perder áreas de producción", posteriormente al ver los resultados lo aplicaron en sus parcelas.

En otra área, se halló que si bien para los agricultores el mejor suelo para el Cacao, es el del paisaje de vega, pudieron establecer comparaciones en diferentes calidades del recurso suelo en el paisaje de lomerío. Uno de los mitos que los campesinos tenían en 
su conocimiento, es que el recurso suelo aun trabajado o aprovechado con cierto cultivo, después de un periodo de descanso de "mas o menos 10 años, la vegetación ha permitido que se recupere $y$ se puede volver a aprovechar". Esto quedó en entredicho al analizar las propiedades físicas y biológicas del suelo en sus lotes que habían sido utilizados en cultivo de coca por espacio de cinco años y que llevaba 10 años en descanso, todos creían que ya se podía volver a aprovechar, pues había crecido una vegetación de porte alto propia de un rastrojo hecho. Al realizar la identificación de organismos vivos solo fue posible encontrar tres coleópteros, tomando 15 puntos de referencia. Esto permitió entender que el grave impacto del uso de los productos de síntesis química en el recurso y la biota y fauna asociada a este permanecía en el tiempo.

En el área de injertación, se halló que aunque este era el tema de menos conocimientos previos también constituía el mayor interés. A partir de las ECAs, se logró que los campesinos conocieran y manejaran los conceptos de patrón, yemas y varetas portayemas y tipos de injertos entre otros. Así mismo, adquirieran la habilidad para diferenciar entre una plantación híbrida y una clonal y las principales las características diferenciales entre clones. Sin embargo, la falta de agilidad y exceso de fuerza al injertar por parte de los adultos, hizo que muchos no lograran el prendimiento esperado en los ejercicios prácticos encomendados como tareas. Aquí el apoyo de los jóvenes fue fundamental, pues para ellos si fue más fácil el desarrollo de las técnicas de injertación.

\section{Lecciones aprendidas}

- Lo ideal es manejar ciclos de formación por perfiles del grupo, esto se puede disgregar siempre y cuando se cuente con un número significativo de asistentes. En el caso de las ECAs en el Caguán por la dispersión de los asistentes, se hicieron grupos por facilidad de acceso, sin embargo, los grupos eran bastante heterogéneos, pues mientras algunos asistentes nunca habían tenido cacao y querían establecer, otros ya tenían plantaciones en crecimiento y otros ya estaban en punto final de su producción, esto hace en algunos temas pierdan interés para cierto tipo de asistentes, mientras que para otros sean los más relevantes. La lección aprendida en esta parte tiene que ver entonces con establecer dentro de las reglas del juego para el funcionamiento de la ECA la asistencia, solidaridad y respeto para todos los módulos de estudio independiente del nivel de conocimiento del tema. De igual forma se pudo aprovechar el mayor conocimiento previo de los agricultores que tenían plantaciones a nivel del compartir su experiencia, logros y fracasos.

- No se debe generalizar avanzar un tema por encuentro, módulos como por ejemplo plagas y enfermedades son demasiado amplios y no se alcanzan a abordar en las ocho horas de trabajo. Esto fue necesario replantearlo con los productores, en razón a la poca disponibilidad de tiempo por sus múltiples tareas en los predios.

- Es necesario que la ECA vaya acompañada de un proceso más frecuente de acompañamiento en campo no solo en el corto sino en el mediano y largo plazo, para evaluar la adopción del aprendizaje, 
pues es diferente que los agricultores lo ensayen a que en realidad lo adopten en su predio. De igual forma las condiciones en los predios son particulares y algunos métodos son cambiados por ellos, funcionales o no bien vale la pena revisarlos y a partir de ahí construir nuevas bases de conocimiento. - Aunque teóricamente las ECAs tienen duración un año o lo que dure el ciclo del cultivo, estas formas de aprendizaje generan otro tipo de situaciones en las comunidades que van más allá de lo académico-productivo y están relacionadas con la unión de la vereda y el despertar interés por pensar no solo individualmente sino de manera colectiva, muchas veces por el diseño de la ECA misma donde se debe dirigir solo al cultivo, esos espacios y oportunidades no se identifican y no se aprovechan como debiera, pues son estratégicas a la hora de tomar decisiones, entre ellas, de nuevas adopciones tecnológicas. - Las ECAs en el Caguán fueron un pretexto para vincular a las familias al cultivo del cacao, en esta zona de trabajo, es muy frecuente jefes de hogar solo con los hijos, o madres cabeza de familia con sus pequeños, los cuales eran llevados por sus padres alas ECAs, por ello se hizo necesario hacer un plan de atención dentro de las ECAs lúdico de aprendizaje pero dirigido a los niños que llegaban a los encuentros, con el fin de que sus padres estuvieran concentrados en el ejercicio.

- En las ECAs, se trabajó con olla comunitaria, para ello al inicio se delegaban unas personas que se encargaban de la preparación de alimentos, sin embargo eran asistentes a los encuentros que se quedaban sin la información, esta estrategia se cambió y entre todos los asistentes se llegaba más temprano al encuentro y se adelantaba el almuerzo y posteriormente si se iniciaba la ECA. - Las ECAs generalmente se hacen con dos facilitadores, es necesario una tercera persona dedicada específicamente a la sistematización de la información de percepción social, el sentir de los asistentes, el antes y el después, las cosas que no se pueden ver a simple vista.

\section{CONCLUSIONES}

La evaluación de la experiencia en el Caguán permitió una reflexión metodológica al interior del equipo de investigación, pues si bien fue y continúa siendo una investigación con enfoque mixto, multidisciplinaria, reconoce que la especificidad del conocimiento con que son formados los profesionales aunque se quiera hacer la transición a modelos más interdisciplinarios hay un riesgo alto que en el camino se pierdan elementos de la integralidad y aunque se trabaje con el conocimiento campesino, se continúe pensando solo en la parcela, no en la investigación y la generación de conocimiento para la sociedad y el territorio.

El proceso de formación en la zona del Caguán con las ECAs de Theobroma cacao L., permitió que las familias se interesaran más por el cultivo y vislumbraran su importancia económica y la necesidad de incorporar tanto prácticas culturales idóneas para 
mejorar sus producciones como mejor organización social para volver a posicionar su producto.

Los asistentes a las ECAs, comprendieron que no es solo el mejoramiento genético de sus plantaciones, como solución a sus problemas productivos, sino que esto va acompañado de una serie de elementos técnicos, como la adecuada selección de terreno, el plan de fertilización, el manejo integrado de plagas y enfermedades y las podas, cuya responsabilidad mayor, está en mano de ellos mismos.

Las ECAs deben ser un proceso de aprendizaje no menor a tres años en el caso del Cacao, sobre todo cuando el perfil de los integrantes del grupo es tan heterogéneo y su localización es excesivamente dispersa, o en su defecto se deben contar con mayores recursos económicos que permitan cubrir los gastos de desplazamiento de los integrantes y reunirlos por perfil del integrante.

La ECA, es una herramienta valiosa en procesos de educación popular pues propende por un aprendizaje social, por tanto no debe limitarse a un ejercicio de extensión rural en lo meramente técnico, esto se profundizará en los siguientes niveles de formación en la ECA-Caguán.

Si bien la ECA es como su nombre lo dice Escuela de Campo de Agricultores dirigida a adultos, el papel de lo que piensan los hijos de los campesinos desde la niñez o la adolescencia acerca del cultivo con el que fueron criados o en el que su familia pretende ingresar, es de vital importancia para el sostenimiento del mismo en el tiempo, de igual forma para identificar los retos que se tendrán que afrontar en términos de adopción y desadopción.

\section{REFERENCIAS BIBLIOGRÁFICAS}

- ARDON, M. _. Escuelas de campo para agricultores (ECAs), en el desarrollo rural una propuesta metodológica coherente. 11 pgs.

- FAO. 2005. Las escuelas de campo para agricultores (ECAs) en el PESA Nicaragua. Una experiencia significativa de extensión para contribuir a la seguridad alimentaria en Nicaragua. Ministerio de Asuntos Exteriores y de Cooperación. Agencia Española de Cooperación. Tegucigalpa, Honduras. 27 pgs.

- ICP. 2006. Guía de Escuelas de Campo de Agricultores ECAs como estrategia de gestión de calidad de Café en el Norte de Perú. Lima, Perú. 58 pgs.

- LUDOJOSKI, R.L. 1972. Andragogía o Eduación del Adulto. Colección la Educación en el tiempo. Instituto de profesorado Verbo Divino Rafael Calzada. Buenos Aires. 275 pgs.

- ICP (Internacional Coffe Partners). 2006. Guía de Escuela de Campo de Agricultores - ECAs. Como estrategia de gestión de calidad de café en el norte de Perú. 58 Págs. 
- IGAC (Instituto Geográfico Agustín Codazzi). 1993. Aspectos ambientales para el ordenamiento territorial del occidente del departamento del Caquetá. 3v.

- CIP (Centro Internacional de la Papa). 2009. Sistematización de las Escuelas de Campo de Agricultores (ECAs) EN Andahuaylas. Lima, Perú. 44 Págs.

- IICA (Instituto Interamericano de Cooperación para la Agricultura). 2010. Escuelas de Campo para Agricultores de Café y Cacao. Experiencias y lecciones aprendidas en la Selva Central. Lima, Perú. 44 Págs.

- IICA (Instituto Interamericano de Cooperación para la Agricultura). 2007. Escuelas de Campo para Agricultores de Cacao en el Perú. Experiencias, resultados y lecciones aprendidas. Lima, Perú. 46 Págs.

- IICA (Instituto Interamericano de Cooperación para la Agricultura). 2006. ACCESO (Programa Oportunidad de Apoyo a Exportaciones de Cacao en Países Andinos). Taller regional andino de transferencia tecnológica en cacao. Lima, Perú. 104 Págs.

- PUMISACHO. M., SHERWOOD. S. 2005. Guía metodológica sobre ECAs -Escuelas de Campo de Agricultores. Instituto de Investigaciones Agropecuarias- INIAP. Centro Internacional de la Papa- CIP. World Neighbor. Estación Experimental Santa Catalina. Quito- Ecuador. 184 pgs.

- USAID (United States Agency International Development). 2009. Guía para la implementación del Modelo Metodológico Escuelas de Campo de Agricultores USAID del Pueblo de los Estados Unidos de Norte América y Acción Social. 123 Págs.

- GALLAGHER, K. D. 1999. Farmers Field Schools (FFS): A Group Extension Process Based on Adult Non-Formal Education Methods. 25 Págs.

\section{REFERENCIA ELECTRÓNICA}

- actrav, Apartados de la Teoría del aprendizaje social. Bandura 1977. Disponible En: hptt:// www.actrav.itcilo.org/courses/2008/A201019/resources/.../bandura.doc

Fecha de consulta: 05 marzo de 2013.

\section{Agradecimientos}

Los autores agradecen al Comité de Cacaoteros de Remolinos del Caguán y Suncillas - CHOCAGUÁN, al Comité de Cacaoteros del Cristal-CHOCOCRISTAL, La Parroquia San Isidro Labrador, El Centro Educativo los Cristales, los amigos de Cuba, Palmichales y el Cristal, todas y cada una de las personas que asistieron a las Escuelas de Campo y recibieron al equipo de la Universidad de la Amazonia cada sesión de trabajo, a ellos nuestra gratitud, cariño y respeto. A cada uno de nuestros estudiantes que caminan hacia la transición agroecológica en otras formas de ver el mundo donde lo espiritual se convierte en parte fundamental de la construcción del conocimiento científico. 
Potencial conflicto de intereses: la presente publicación se hace con permiso de los involucrados.

Fuentes de financiación: la presente investigación fue posible gracias a la contribución económica del Departamento Administrativo de Ciencia, Tecnología e Innovación COLCIENCIAS, a través del convenio 719-2012 entre la Fiduciaria Bogotá, la Universidad de la Amazonia y el Comité de Cacaoteros de Remolinos del Caguán y Suncillas CHOCAGUAN.

1. Ingeniera Agroecóloga, Universidad de la Amazonia. M Sc. En Ambiente y Desarrollo, estudiante Doctorado en Antropología Universidad del Cauca. Docente Universidad de la Amazonia.

2. Ingeniero Agroecólogo, Universidad de la Amazonia. M Sc. En Agricultura Ecológica, estudiante Doctorado en Ciencias Agrarias, Docente Universidad de la Amazonia.

Para citar este artículo: Sánchez Castillo, V. \& Gamboa Tabares, J. A. (2014). Escuelas de campo de agricultores de Theobroma cacao I. en el Bajo Caguán (experiencia, resultados y lecciones aprendidas). Revista Luna Azul, 38, 231-251. Recuperado de http://lunazul.ucaldas.edu.co/index.php?option=content\&task=v iew\&id=904 